“C 2017 IEEE. Personal use of this material is permitted. Permission from IEEE must be obtained for all other uses, in any current or future media, including reprinting/republishing this material for advertising or promotional purposes, creating new collective works, for resale or redistribution to servers or lists, or reuse of any copyrighted component of this work in other works." 


\title{
Accurate Copper Loss Analysis of a Multi-winding High-frequency Transformer for a Magnetically-coupled Residential Micro-grid
}

\author{
Mohammad Jafari, member IEEE, Zahra Malekjamshidi, and Jianguo Zhu, Senior member, IEEE \\ University of Technology Sydney, NSW 2007, Australia
}

\begin{abstract}
Improvements in characteristics of magnetic materials and switching devices have provided the feasibility of replacing the electrical buses with high frequency magnetic links in micro-grids. This effectively reduces the number of voltage conversion stages, and the size and cost of the renewable energy system. It also isolates the converter ports which increases the system safety and facilitates bidirectional power flow and energy management. To design the magnetic link optimally, an accurate evaluation of copper loss of the windings considering both current waveforms and parasitic effects is required. This paper studies the accurate copper loss analysis of a three-winding high-frequency magnetic link for residential micro-grid applications. Due to the non-sinusoidal nature of the voltage and currents, the loss analysis is carried out on a harmonic basis taking into account variations of phase shift, duty ratio and amplitude of wave-forms. The high frequency skin and proximity effects are taken into account. The maximum and minimum copper loss operating points of the converter and their dependency on the phase shift and duty ratio of the waveforms are studied and simulation results are presented.
\end{abstract}

Index Terms-Copper loss, harmonics, high-frequency magnetic link, multi-winding transformer, phase-shift, proximity, skin effects

\section{INTRODUCTION}

$\mathbf{H}$ IGH-FREQUENCY magnetic links have attracted a lot of research interest due to their application in the integration of renewable energy resources into the grid. The magnetic link is a multi-winding high frequency transformer which provides bidirectional paths for magnetic fluxes and power flows between different ports. As a feasible replacement for conventional electrical buses, it can be used to integrate renewable energies in smart grids. It can reduce effectively the number of conversion stages and improve the conversion efficiency using lossless switching devices and modern soft magnetic materials. Other advantages are galvanic isolation and bidirectional power flow capability between the converter ports [1]-[6]. Fig.1 illustrates two renewable energy systems with and without the magnetic link. The dashed lines show that the power flow path from PV array to the battery has been reduced effectively with the use of the magnetic link. Therefore employing the magnetic link can reduce the number of voltage conversion units, increase the system efficiency and reduce the system's size and cost. To optimally design the magnetic link, the winding loss should be accurately evaluated considering non-sinusoidal effects of the voltage and current waveforms [7].

The high frequency time-varying harmonics of non-sinusoidal currents produce the well-known skin and proximity effects which cause a non-uniform distribution of the current in the wire area [7],[8]. Due to the dependency of these effects to operating frequency, a frequency-dependent resistance model is used to analyze the copper loss [9]-[13].To reduce the copper loss, Litz wires are recommended for high frequency applications [9]-[14]. The loss evaluation of Litz wire windings is mainly based on either numerical [9], [10] or analytical [11]-[19] field analysis. The numerical methods provide more accurate results although they are relatively complicated and computationally expensive. On the contrary, the analytical methods are easy for design purposes and present good accuracy where Litz wire strand radius remains below the skin depth [7], [22]. The analytical methods in turn can be divided into three groups [7]. A majority of works are based on Dowell's work in 1966 on loss analysis of foil and round conductors using the Maxwell's equations in Cartesian coordinates [11]. They have extended the Dowell's method for foil conductors to the Litz wire windings of the same cross-section area and have applied a porosity factor to the resultant equation to increase the accuracy [12],[13]. The second approach known as Ferreira method is based on exact field solution of stand-alone round conductor using Bessel functions [14]. The analysis was later extended to a complete winding [15], [16] and an expression was introduced using modified Bessel functions to improve the accuracy of the method in high frequency applications [17]. In the third group, conduction loss is calculated using complex conductor permeability [18]. The method provides a good accuracy although it is complicated to determine the real and imaginary parts of the winding permeability [7]. The first two methods are widely used in the literature and their accuracy is compared [16], [19]. Both methods provide good accuracy especially where strand diameter is much lower than the skin depth [7], [13], [16]. A detailed study on copper loss analysis of buck and fly-back dc-dc converters under variable duty ratio based on the first analytical method has been presented recently [20],[21] although none of these studies deal with the multiple winding transformer and phase shifted waveforms. In [8] the copper loss of a dual active bridge (DAB) bidirectional converter is studied considering the effects of phase shift of voltages applied to the transformer.

In this paper, research in [8] is extended to the case of threewinding magnetic link while both amplitude and phase shifts are variable. The maximum and minimum loss conditions are defined for two common micro-grid operation modes known as $\mathrm{DAB}$ and triple active bridge (TAB). 


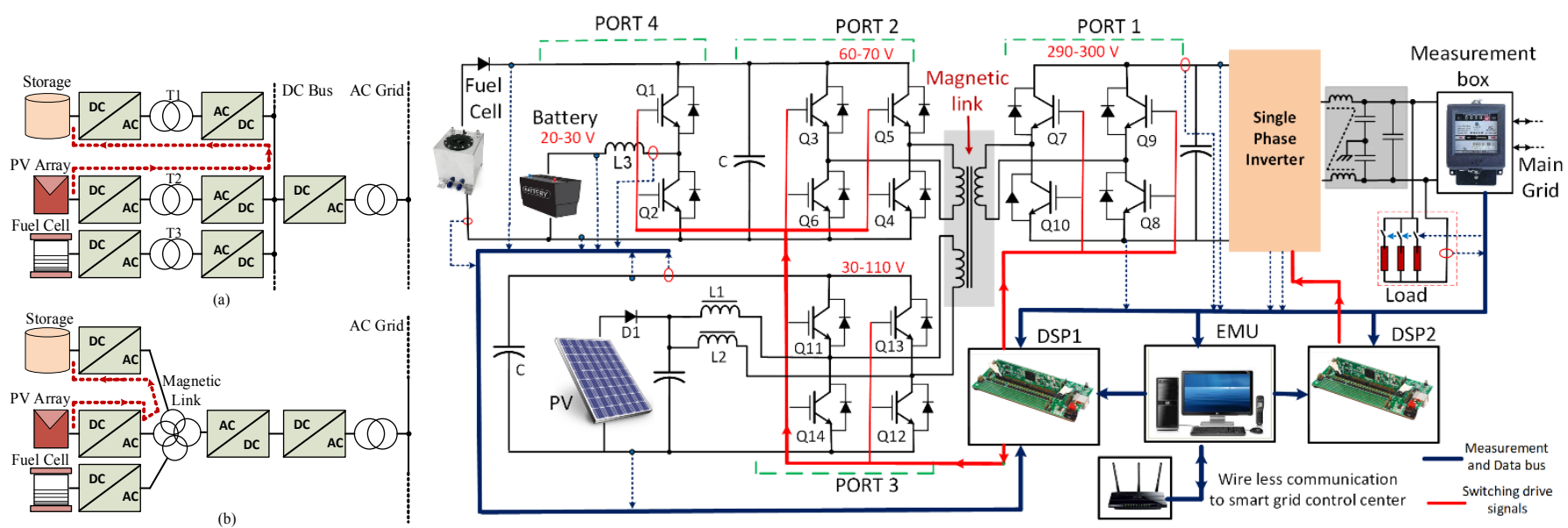

Fig. 1. Comparing two renewable energy systems,

(a) without magnetic link, and (b) with magnetic link.

Fig. 2. Schematic of the proposed residential micro-grid

\section{ApPlication of Magnetic LinK In RESIDENTIAL}

\section{MICRO-GRID}

The proposed micro-grid topology including three-winding magnetic link is illustrated in Fig.2. As can be seen in the figure, the proposed topology includes four ports. Port one, two and three are linked together through the magnetic link and form a TAB phase shift converter. They are used to convert the dc voltage of fuel cell, PV and inverter bus to a high frequency ac voltage fed to the windings of the magnetic link. Port four links the battery to the fuel cell bus to store the surplus energy of the system and release it when is required. It also operates in standby mode to balance the voltage of the fuel cell bus in transients due to slow dynamic response of fuel cell. The system is connected to the residential load and grid via a single phase bidirectional inverter and is designed to supply a $5 \mathrm{~kW}$ residential load. Two digital signal processors (TMS320F28335) are used to control the dc-dc converters and inverter at the device level. The system level control and energy management, considering both long term and short term load and energy forecasts is performed in a PC system. The regional distribution network control center communicates with energy management unit (EMU) via internet (through either cable or wireless).

To control the power flow from PV and fuel cell ports to the inverter, the waveforms of the voltages of port two and three are shifted for leading phase angles of $\varphi_{21}$ and $\varphi_{31}$ compared to port one to send power to the inverter. In the PV port, switching devices of H-bridge dc-ac converter are shared with an interleaved current-fed boost converter. The interleaved boost converter is used to maintain MPPT through duty ratio control while dc-bus voltage regulation is carried out using phase shift angle $\varphi_{31}$. The voltage on the PV port changes reversely with duty ratio to keep the equal volt-seconds on all three windings of the magnetic link and guarantees the zero voltage switching (ZVS) operation of TAB converter ports. The proposed microgrid can operate in various operation modes based on the power flow directions and active ports.

\section{COPPER LOSS ANALYSIS METHOD}

Due to the linear behavior of copper, conduction loss analysis is carried out based on the harmonic contents of the currents in the windings of the magnetic link [7], [8], [20], [21]. Therefore, the total copper loss can be defined by integration of resultant loss of each harmonic component of the current as following

$$
P_{C u}=\sum_{n=1}^{h} P_{C u, n}
$$

where $P_{C u, n}$ is the resultant copper loss of $n$th harmonic and $h$ is the number of harmonics that are effective in loss analysis.

The skin and proximity effects on the windings resistance can be considered separately for copper loss analysis of each harmonic due to their orthogonality principle [23]. Their effect is modelled as a frequency-dependent resistance for each harmonic in the case of non-sinusoidal currents. Therefore, the resultant loss of the $n$th harmonic, $P_{C u, n}$, is calculated by

$$
P_{C u, n}=\left(\frac{I_{n}}{\sqrt{2}}\right)^{2} R_{a c, n}
$$

where $R_{a c, n}$ is the ac resistance of the Litz-wire winding for $n$th harmonic and $I_{n}$ the peak value of $n$th harmonic of the nonsinusoidal current in the winding. To calculate the copper loss using (1) and (2) for the proposed magnetic link, the analytical methods of calculating equivalent ac resistance $R_{a c, n}$, and amplitude of each harmonic $I_{n}$, are discussed in the following sections.

\section{HARMONIC BASED ANALYSIS OF THE CURRENTS IN THE MAGNETIC LINK}

The leakage inductances of the windings of the magnetic link are the main elements of the power transfer between the ports in the proposed TAB converter. A T-model configuration of the three-winding magnetic link can be obtained by referring leakage inductances of windings two and three to winding one as reference (magnetizing inductance is neglected as it has no effect on power transfer). The resultant model then can be transformed to the equivalent $\Delta$-model to obtain the simplified TAB converter model as presented in Fig.3 [3],[6]. On the other hand, the dc-bus and $\mathrm{H}$-bridge converters are replaced with an equivalent square 
wave ac source. The leading phase shift angles, $\varphi_{21}$ and $\varphi_{31}$, are assumed between voltages of ports two and three to port one (selected as reference port) as presented in Fig.4. This is because power transfers from fuel cell and PV ports to the inverter port. A zero-voltage angle $\Omega$, is introduced in the PV port ac voltage $v_{3}$, to maintain the MPPT. Therefore, the de voltage on PV port $V_{3}$, should be varied accordingly to maintain the ZVS operation.

$$
V_{3}=\frac{1}{1-(\Omega / \pi)} V_{3-\min }
$$

where $V_{3}$ is the actual value and $V_{3-\min }$ is the minimum value of voltage on PV port. The duty ratio $D$, can be defined from

$$
D=(1-\Omega / \pi)
$$

In this paper, the duty ratio of fuel cell and inverter ports is kept constant $(D=1, \Omega=0)$ due to the constant voltage of their buses.

The harmonic based analysis of non-sinusoidal currents has been widely used for copper loss analysis of transformers [8], [20], [21]. To calculate the copper loss in the proposed magnetic link, currents in the windings are analyzed using the equivalent $\Delta$-model. Therefore, fundamental-harmonic phasor diagram of the currents and voltages in the equivalent $\Delta$-model, presented in Fig.5 is used as a reference in the following analysis.

The high frequency square wave voltages illustrated in Fig.4, are represented based on their Fourier series as

$$
\begin{gathered}
v_{1}(t)=\sum_{n=1}^{\infty} V_{1, n} \sin (n \omega t) \\
v_{2}(t)=\sum_{n=1}^{\infty} V_{2, n} \sin \left(n\left(\omega t+\varphi_{21}\right)\right) \\
v_{3}(t)=\sum_{n=1}^{\infty} V_{3, n} \sin \left(n\left(\omega t+\varphi_{31}\right)\right)
\end{gathered}
$$

where $V_{k, n}$ is the amplitude of the $n$th harmonic of the voltage across winding $k$. For port one and port two due to their constant amplitude, we have

$$
V_{k, n}=\frac{2 V_{k}(1-\cos (n \pi))}{n \pi}, \quad k=1,2
$$

For port three, due to the variable amplitude and duty ratio, it can be calculated by

$$
V_{3, n}=\frac{2 V_{3-\min } \cos (n \Omega / 2)(1-\cos (n \pi))}{n(\pi-\Omega)}
$$

As can be seen in the equivalent model, $i_{1}=i_{13}-i_{21}, i_{2}=i_{21}-i_{32}$ and $i_{3}=i_{32}-i_{13}$. Therefore, to analyze the current in the winding one $i_{1}$, the currents $i_{21}$ and $i_{13}$ should be calculated by using voltage across inductances $L_{21}$ and $L_{13}$. The voltage $v_{21}$ can be calculated using (5) by

$$
v_{21}(t)=v_{2}(t)-v_{1}(t)=\sum_{n=1}^{\infty} V_{21, n} \cos \left(n \omega t+\alpha_{n}\right)
$$

where $V_{21, n}$ is the amplitude of $n$th harmonic of $v_{21, n}$ and is defined from

$$
V_{21, n}=\sqrt{V_{1, n}^{2}+V_{2, n}^{2}-2 V_{1, n} V_{2, n} \cos \left(n \varphi_{21}\right)}
$$

and the phase angle of $n$th harmonic $\alpha_{n}$, is calculated using

$$
\alpha_{n}=\tan ^{-1} \frac{V_{2, n} \sin \left(n \varphi_{21}\right)}{V_{2, n} \cos \left(n \varphi_{21}\right)-V_{1, n}}
$$

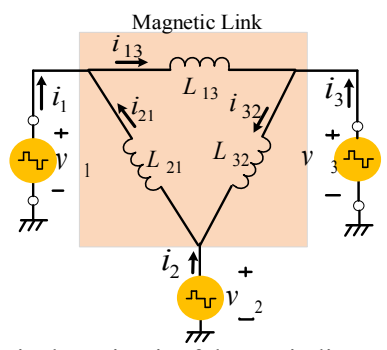

Fig. 3. The $\Delta$-model equivalent circuit of three winding magnetic link.

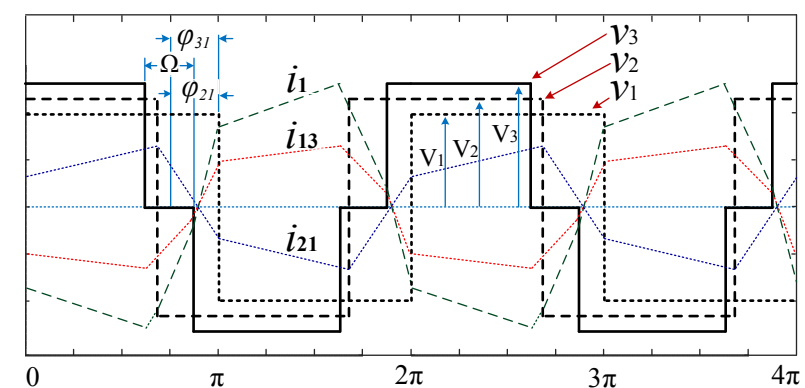

Fig.4. Voltage and current wave forms related to current $i_{l}$ in winding one.

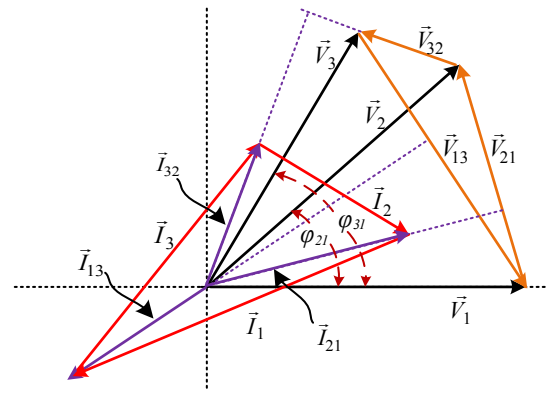

Fig.5. Fundamental phasor diagram of the TAB converter

The current $i_{21}$ can then be calculated taking into account $\pi / 2$ as inductive phase shift angle by

$$
i_{21}=\sum_{n=1}^{\infty} I_{21, n} \sin \left(n \omega t+\alpha_{n}-\frac{\pi}{2}\right)
$$

where $I_{21, n}$ is the amplitude of the $n$th harmonic of $i_{2 l, n}$ and can be calculated by

$$
I_{21, n}=\frac{V_{21, n}}{\left(2 \pi n f L_{21}\right)}
$$

The current $i_{13}$ in the winding $L_{13}$ is defined similar to $i_{21}$ from (13) considering $\varphi_{31}$ as the phase shift angle between $v_{3}$ to $v_{1}$, $I_{13, n}$ as the amplitude and $\left(\beta_{n}-\pi / 2\right)$ as the phase angle of $n$th harmonic.

$$
i_{31}=\sum_{n=1}^{\infty} I_{31, n} \sin \left(n \omega t+\beta_{n}-\frac{\pi}{2}\right)
$$

Finally, the $n$th harmonic of the current in the source $v_{l}$ and in the winding one of the magnetic link $i_{1, n}$ (refer to Fig.3) can be calculated using (11) and (13) by

$$
i_{1, n}(t)=i_{13, n}-i_{21, n}=I_{1, n} \sin \left(n \omega t+\psi_{1, n}\right)
$$

where $I_{l, n}$ and $\psi_{l, n}$ are the amplitude and the phase angle of $n$th harmonic of $i_{l}$ respectively and can be determined by

$$
\begin{array}{r}
I_{1, n}=\sqrt{I_{13, n}^{2}+I_{21, n}^{2}-2 I_{13, n} I_{21, n} \cos \left(n\left(\beta_{n}-\alpha_{n}\right)\right)} \\
\psi_{1, n}=\tan ^{-1}\left(\frac{I_{13, n} \sin \left(n \beta_{n}\right)-I_{21, n} \sin \left(n \alpha_{n}\right)}{I_{13, n} \cos \left(n \beta_{n}\right)-I_{21, n} \cos \left(n \alpha_{n}\right)}\right)
\end{array}
$$


The amplitude of each harmonic of the current in winding two, $I_{2, n}$ and the phase angle $\psi_{2, n}$ and in winding three, $I_{3, n}$ and $\psi_{3, n}$ can be calculated in a similar way. It can be seen from (5) and (7) that $v_{1}, v_{2}$ and $v_{3}$ and consequently the resultant current in the windings only contain the odd harmonics. Once the harmonic content of the current in the windings $\left(i_{1, n}, i_{2, n}\right.$ and $\left.i_{3, n}\right)$ is determined, the ac resistance factor of each winding $R_{a c, n}$, related to each harmonic term should be defined for loss analysis. The next section provides the details of calculating ac resistance.

\section{CAlCUlation of AC Resistance}

The skin and proximity effects are considerable in medium and high-frequency applications. To reduce these effects the stranded, twisted insulated conductors known as Litz wires should be used. Considering these high frequency effects, the ac resistance of a Litz wire winding is a function of frequency while the dc resistance is relatively constant and for each winding can be calculated by

$$
R_{d c}=\frac{4 N l_{s} \rho_{C u}}{n_{s} \pi d_{s t r}^{2}}
$$

where $N$ is the turns number of winding, $l_{s}$ the average length of one turn, $\rho_{c u}$ the resistivity of copper, $d_{s t r}$ the strand diameter and $n_{s}$ the number of strands. The skin and proximity effect losses related to each harmonic of the current can be modeled through a frequency-dependent resistance, $R_{a c, n}$ [7]-[17]. The ratio of $R_{a c, n}$ to $R_{d c}$ known as the ac resistance factor $F_{r, \mathrm{n}}$, can be defined as

$$
F_{r, n}=\frac{R_{a c, n}}{R_{d c}}
$$

In the case of using Litz wires the skin and proximity effects appear in both strand and bundle levels [13]. The bundle level proximity effect can be reduced efficiently by twisting; however, bundle level skin effect needs more complex constructions [13], [22]. On the other hand the strand level proximity effect is more considerable compared with skin effects especially in the case of increasing number of layers [22]. The skin and proximity effects analysis in Litz wires are carried out based on two best-known analytical methods, the Dowell method and Ferreira method. In the first method, the round conductors are replaced with square conductors of the same area and then an equivalent foil conductor the same as the one that Dowell analyzed is found. The one dimensional field analysis is applied to the foil to find the losses [11]-[13]. The second approach is based on the field analysis of a single round conductor using well-known Bessel functions and some of its applications are proposed by Ferreira [14]-[17]. Both methods provide similar results and accuracy for small penetration ratios [8] although it has been shown that the second method provides more accurate results in high frequency applications [17]. In this paper the first method is used for our special case due to its simplicity, relatively low switching frequency $(10 \mathrm{kHz})$ and penetration ratio. In this method (proposed in 1966), the skin and proximity effect losses of an isolated solid round conductor were evaluated using an exact solution of the internal and external magnetic fields [14] and further developed by Ferreira in 1990 [22], [23]. The closed form expression considering orthogonality of skin and proximity effects can be written as

$$
F_{r, n}=\frac{\gamma_{n}}{2}\left[\tau_{1, n}-2 \pi \frac{4\left(m^{2}-1\right)}{3} \tau_{2, n}\right]
$$

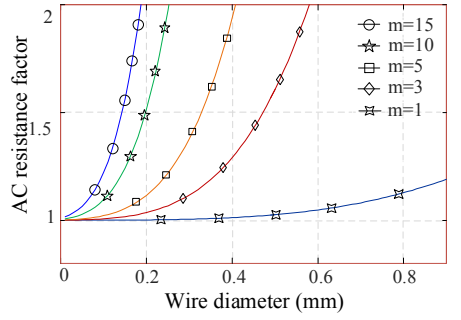

(a)

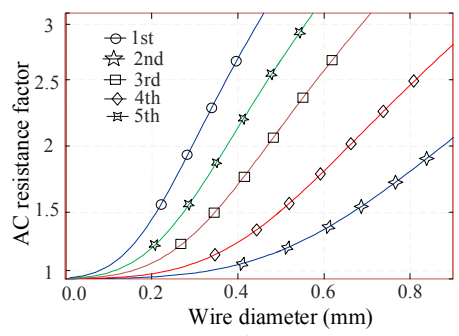

(b)
Fig.6. Ac resistance factor as a function of wire diameter for: (a) different number of winding layers, and (b) various harmonics of the current in windings of the magnetic link.

with

$$
\begin{gathered}
\tau_{1, n}=\frac{\operatorname{ber}\left(\gamma_{n}\right) b e i^{\prime}\left(\gamma_{n}\right)-\operatorname{bei}\left(\gamma_{n}\right) b e r^{\prime}\left(\gamma_{n}\right)}{\operatorname{ber}^{\prime}\left(\gamma_{n}\right)^{2}+\operatorname{bei}^{\prime}\left(\gamma_{n}\right)^{2}} \\
\tau_{2, n}=\frac{\operatorname{ber}_{2}\left(\gamma_{n}\right) \operatorname{ber}^{\prime}\left(\gamma_{n}\right)+\operatorname{bei}_{2}\left(\gamma_{n}\right) b e i^{\prime}\left(\gamma_{n}\right)}{\operatorname{ber}^{\prime}\left(\gamma_{n}\right)^{2}+\operatorname{bei}^{\prime}\left(\gamma_{n}\right)^{2}}
\end{gathered}
$$

where ber and bei are the real and imaginary parts of the first kind Bessel function and $\gamma_{n}$ is the round conductor penetration ratio of the $n$th harmonic and is defined as

$$
\gamma_{n}=\frac{d}{\delta_{n} \sqrt{2}}
$$

\section{Copper Loss Evaluation Of THE MAgNetic LinK}

This section reviews the loss calculation of the magnetic link considering all effective elements. As can be seen in (19)-(21), the harmonic frequency, strand diameter and number of winding layers have major effects on the ac resistance factor and consequently the copper loss. Fig.6 (a) illustrates the effect of increasing the number of winding layers on the ac resistance factor. As can be seen the ac resistance of the wire decreases by reducing number of windings layers and using strands of less diameter. Fig.6 (b) shows the ac resistance factor, $F_{r, n}$ for the five major harmonics of the converter currents. It can be seen that the ac resistance factor reduces for lower harmonic orders and strand diameters. Therefore, reducing operation frequency reduces not only the switching loss but also the winding losses in the converters.

\section{A. Calculation of maximum copper loss}

The maximum copper loss is important in the optimal design and thermal analysis of the magnetic link. According to the power flow equation of $\mathrm{TAB}$ converter, maximum power flow and $r m s$ current in the magnetic link take place when fuel cell and PV ports are transferring maximum power $\left(\varphi_{21}=\pi / 2, \varphi_{31}=\pi / 2\right)[3]$ [7]. On the other hand, when PV bus voltage $V_{3}$, is maximized and duty ratio $D$, is minimized, the amplitude of the fundamental harmonic of the current in winding three increases and raises the loss. Therefore, duty ratio is equal to its minimum value when copper loss in winding three is maximized $(D=0.25$ and $\Omega=3 \pi / 4$ in this case). The power losses of all windings were analytically calculated for the specified operation point and are integrated to define the maximum copper loss of the magnetic link. Fig.7 illustrates the calculated value of ac resistance $R_{a c, n}$, amplitude of harmonic $I_{n}$, and the resultant copper loss $P_{c u, n}$ of each harmonic of the current in the windings. 


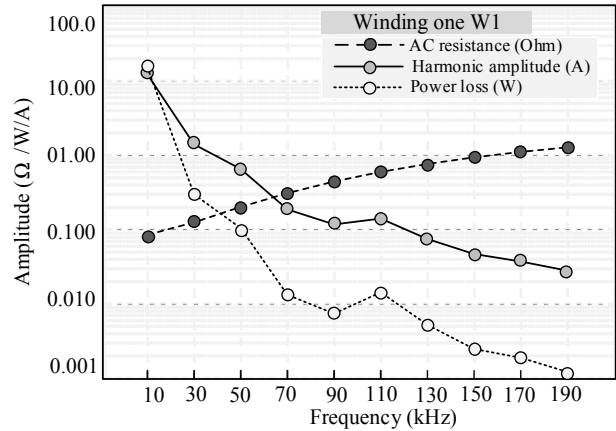

(a)

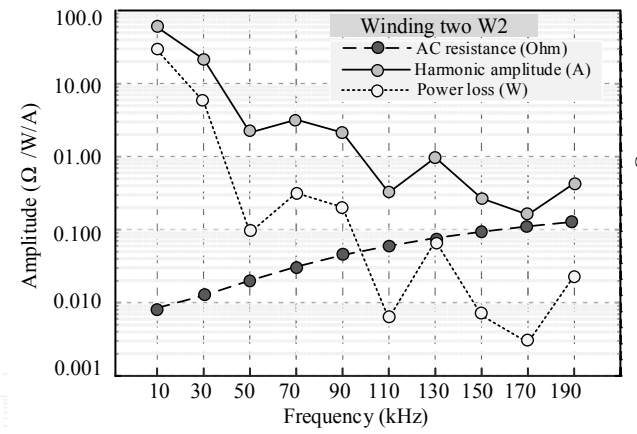

(b)

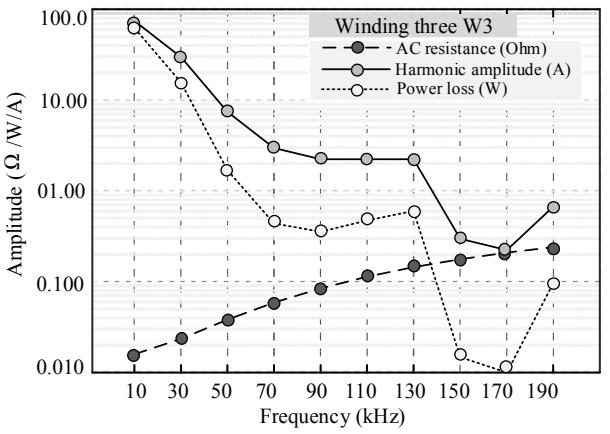

(c)

Fig.7. Calculated value of ac resistance $R_{a c}$, copper loss and harmonic amplitudes of the three windings of the magnetic link for maximum conduction loss condition (a) winding one, (b) winding two, and (c) winding three

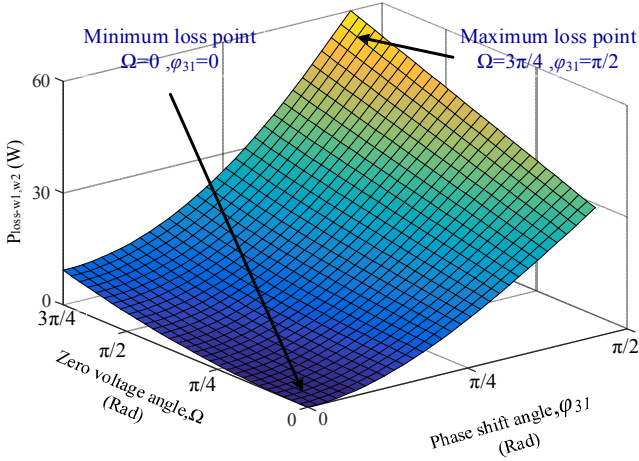

Fig.8. Conduction loss of the windings one and three for zero voltage angle, $\Omega=0$ to $3 \pi / 4$ and phase shift angle, $\varphi_{31}=0$ to $\pi / 2$.

As can be seen the copper loss is decreased with increasing the harmonic orders as the effect of reduction in harmonic amplitude overcomes the increasing ac resistance. The calculated value of copper losses of windings one, two and three using (1) are equal to $15 \mathrm{~W}, 37 \mathrm{~W}$ and $78 \mathrm{~W}$ respectively. Therefore, the maximum copper loss of the magnetic link in this operation mode is $130 \mathrm{~W}$.

\section{B. Loss analysis under phase shift angle and duty ratio variation}

To study the effect of variation of phase shift angle $\varphi_{31}$ and zero voltage angle $\Omega$ of the PV port on the copper loss, two common operation modes have been considered. In the first operation mode only the PV and inverter ports are active and the dc-dc converter operates in DAB mode. The phase shift angle, $\varphi_{31}$ was changed from 0 to $\pi / 2$ and the zero voltage angle $\Omega$, from 0 to $3 \pi / 4$ (assuming that voltage of PV port varies from $30 \mathrm{~V}$ to 120 $\mathrm{V})$. The copper loss of the magnetic link for the entire operation range was calculated according to the method discussed in section III and is presented in Fig.8. It can be seen that in general any increase in the phase shift angle and zero voltage angle increases the copper loss although the effect of phase shift angle is more considerable compared to that of zero voltage angle. Increasing zero voltage angle at higher phase shift angles raises the amplitude of fundamental component of the current in the PV port which results in more effect on the copper loss at higher phase shift angles. As expected the maximum loss takes place at $\varphi_{31}=\pi / 2$ and $\Omega=3 \pi / 4$ which is about $60 \mathrm{~W}$.In the second case the converter operated in the TAB mode and both fuel cell and PV ports were active and transferred power to the inverter port. The fuel cell port was operating with the constant phase shift angle and duty ratio $\left(\varphi_{21}=\pi / 2, \Omega=0\right)$ due to the constant voltage of the bus. At the PV port, the bus voltage $V_{3}$, zero voltage angle $\Omega$ and the phase shift angle $\varphi_{31}$ has been changed similar to the previous stage.

Fig.9 illustrates the overall trend of the currents in the equivalent $\Delta$-model $\left(i_{21}, i_{13}\right.$ and $\left.i_{32}\right)$ and in the windings $\left(i_{1}, i_{2}\right.$ and $i_{3}$ ) with the variation of phase shift angle, $\varphi_{31}$. As can be seen increasing $\varphi_{31}$ raises $i_{31}$ and the power transferred from PV to the inverter while $i_{21}$ remains constant due to the constant value of $\varphi_{21}$. On the other hand, increasing $\varphi_{31}$ reduces $\varphi_{23}$ (as $\varphi_{23}=\varphi_{21^{-}}$ $\left.\varphi_{31}\right)$ and $i_{32}$ and consequently the power transferred from fuel cell port to PV port. Referring to the $\Delta$-model nodal equations $\left(i_{1}=i_{13}-\right.$ $i_{21}, i_{2}=i_{21}-i_{32}$ and $\left.i_{3}=i_{32}-i_{13}\right)$, one can see that when $\varphi_{31}$ increases, $i_{1}$ and consequently copper loss in winding one increases while $i_{2}$ and copper loss in winding two decreases. In winding three, $i_{3}$ decreases for phase shift angles less than $\pi / 4$ and then increases. This is because, for small phase values of $\varphi_{31}$, the power received from port two is more than the power transferred to port one. At $\varphi *_{31}=\varphi_{21} / 2=\pi / 4$ the powers received from port two and transferred to port one are almost equal. Therefore the average power of the port and the current $i_{3}$ are minimized at this point and the copper loss of winding three and the total copper loss of the magnetic link are minimized at this point. As can be seen in Fig.10, the minimum loss point where $\varphi_{31}=\varphi^{*}{ }_{31}$ depends on the $\varphi_{21}$ and always $\varphi^{*}{ }_{31}=\varphi_{21} / 2$ where the received and transferred powers to the port three are equal. The resultant copper loss of all windings and the total loss according to the phase shift angle $\varphi_{31}$, and zero voltage angle $\Omega$, variations are presented in Fig. 11 . 


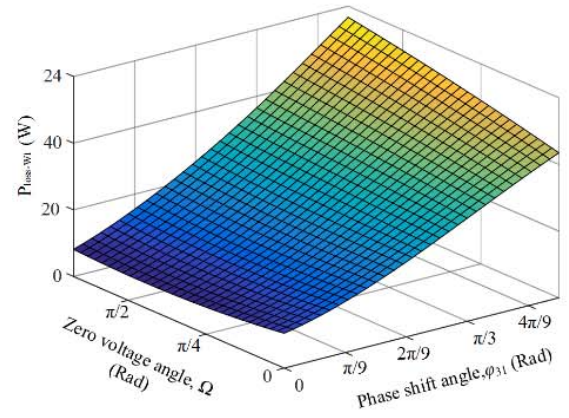

(b)

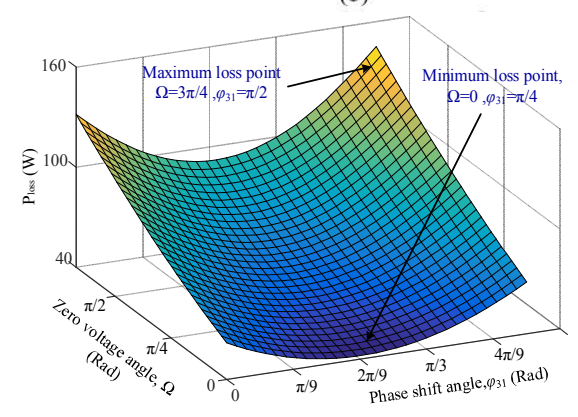

(d)

Fig.11. Conduction loss of the magnetic link for zero voltage angle, $\Omega=0$ to $3 \pi / 4$ and phase shift angle, $\varphi_{31}=0$ to $\pi / 2$. (a). winding one, W1. (b)winding two,W2. (c) winding three, W3, and (d) total magnetic link.

As can be seen in Fig.11 (a)-(b), increasing phase shift angle $\varphi_{31}$, raises the loss in winding one and decreases the average power, current and loss of winding two. As presented in Fig.11(c)-(d), the copper loss of winding three and the total loss are minimized at $\varphi_{31}=\varphi^{*}=\pi / 4$ due to the previous discussions.

\section{CONCLUSION}

An accurate method of copper loss analysis based on the harmonic contents of the excitation waveforms for a multiwinding magnetic link is discussed in this paper. The phase shift angle, duty ratio and amplitude variation of the voltages of the ports on the copper loss have been taken into account. The high frequency skin and proximity effect losses are considered using the ac resistance factor of the Litz wire windings. The total copper loss of the magnetic link and the maximum and minimum copper loss operating points for two cases of $\mathrm{DAB}$ and $\mathrm{TAB}$ modes are calculated analytically.

\section{REFERENCES}

[1] M. Jafari, Z. Malekjamshidi, G. Platt, J. G. Zhu and D. G. Dorrell, "A multi-port converter based renewable energy system for residential consumers of smart grid," in Proc. 41st Ann. Conf. of the IEEE Ind. Electron.Society, Yokohama, IECON 2015, pp. 005168-005173.

[2] Y. M. Chen, Y. C. Liu, and T. F. Wu, "Multi-input dc-dc converter based on the multi-winding transformer for renewable energy applications," IEEE Trans. Ind. Appl., vol. 38, no. 4, pp. 1096-1104, July/August 2002

[3] H. Tao, A. Kotsopoulos, J. L. Duarte and M. A. M. Hendrix, "Transformer-Coupled Multiport ZVS Bidirectional DC-DC Converter With Wide Input Range," IEEE Trans. Power Electron., vol. 23, no. 2, pp. 771-781, March 2008.

[4] M. Jafari, Z. Malekjamshidi, G. Lei, T. Wang, G. Platt and J. Zhu, "Design and Implementation of an Amorphous High-Frequency Transformer Coupling Multiple Converters in a Smart Microgrid,'IEEE Trans.Ind. Electron., vol. 64, no. 2, pp. 1028-1037, Feb. 2017.

[5] M. Jafari, Z. Malekjamshidi, M. R. Islam and J. Zhu, "Modeling of magnetic flux in multi-winding toroidal core high frequency transformers using 3D reluctance network model," 11th IEEE Int. Conf. on Power Electronics and Drive Systems, (PEDS 2015) Sydney, NSW, 2015, pp. 413-418.

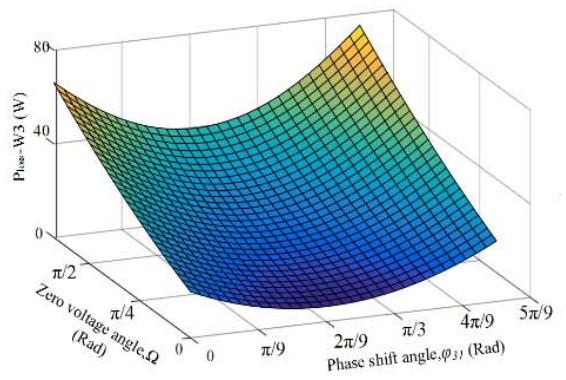

(d)

[6] H. Tao, A. Kotsopoulos, J. L. Duarte, and M. A. M. Hendrix, "Family of multiport bidirectional DC-DC converters," IEE Proceeding Electric Power Applications, vol. 153, no. 3, pp. 451-458, May 2006.

[7] E. L. Barrios, A. Ursúa, L. Marroyo and P. Sanchis, "Analytical Design Methodology for Litz-Wired High-Frequency Power Transformers," IEEE Trans. Ind. Electron., vol. 62, no. 4, pp. 2103-2113, April 2015.

[8] I. Villar, U. Viscarret, I. Etxeberria-Otadui and A. Rufer, "Global Loss Evaluation Methods for Nonsinusoidally Fed Medium-Frequency Power Transformers," IEEE Trans. Ind. Electron., vol. 56, no. 10, pp. 41324140 , Oct. 2009.

[9] H. Hämäläinen, J. Pyrhönen, J. Nerg, and J. Talvitie, "AC resistance factor of litz-wire windings used in low-voltage high-power generators," IEEE Trans. Ind. Electron., vol. 61, no. 2, pp. 693-700, Feb. 2014.

[10] C. R. Sullivan, "Computationally efficient winding loss calculation with multiple windings, arbitrary waveforms, and two-dimensional or threedimensional field geometry," IEEE Trans. Power Electron., vol. 16, no. 1, pp. 142-150, Jan 2001.

[11] P.L.Dowell, " Effects of eddy currents in transformer windings," in Proc. IEE, Vol.113, pp.1387-1394, Aug. 1966.

[12] R. P. Wojda and M. K. Kaizimierczuk, "Winding resistance of litz-wire and multi-strand inductors," IET Power Electron., vol. 5, no. 2, pp. $257-$ 268, Feb. 2012.

[13] C. R. Sullivan, "Optimal choice for number of strands in a litz-wire transformer winding," IEEE Trans. Power Electron., vol. 14, no. 2, pp. 283-291,Mar. 1999.

[14] J. Lammeraner and M. Stafl, Eddy Currents, 1st ed. London, U.K.: Iliffe Books, Ltd., 1966, ch. 7.

[15] M. Bartoli, N. Noferi, A. Reatti, and M. K. Kazimierczuk, "Modeling litz wire winding losses in high-freq uency power inductors," in Proc. IEEE PESC, 1996, pp. 1690-1696.

[16] F. Tourkhani and P. Viarouge, "Accurate analytical model of winding losses in round litz wire windings," IEEE Trans. Magn., vol. 37, no. 1, pp. 538-543, Jan. 2001.

[17] A. W. Lotfi and F. C. Lee, "A high frequency model for Litz wire for switch-mode magnetics," in proc. Ind. Appl. Society Annual Meeting, 1993., Toronto, Ont., 1993, pp. 1169-1175 vol.2.

[18] X. Nan and C. R. Sullivan, "An equivalent complex permeability model for litz-wire windings," IEEE Trans. Ind. Appl., vol. 45, no. 2, pp. 854 860, Mar./Apr. 2009.

[19] J. A. Ferreira, "Improved analytical modeling of conductive losses in magnetic components," IEEE Trans. Power Electron., vol. 9, no. 1, pp. 127-131, Jan. 1994.

[20] D. Murthy-Bellur and M. K. Kazimierczuk, "Harmonic winding loss in buck DC-DC converter for discontinuous conduction mode," IET Power Electron., vol. 3, no. 5, pp. 740-754, September 2010.

[21] D. Murthy-Bellur, N. Kondrath and M. K. Kazimierczuk, "Transformer winding loss caused by skin and proximity effects including harmonics in pulse-width modulated DC-DC flyback converters for the continuous conduction mode," IET Power Electron., vol. 4, no. 4, pp. 363-373, April 2011.

[22] I. Villar, "Multiphysical characterization of medium-frequency power transformers," Ph.D. dissertation, Faculté des Sciences et Techniques de l'Ingénieur, École Polytechnique Fédérale Lausanne, Lausanne, Switzerland, 2010.

[23] J. A. Ferreira, "A Analytical computation of AC resistance of round and rectangular litz wire windings," in IEE Proc. B-Electric Power Applications, vol. 139, no. 1, pp. 21-25, Jan. 1992. 